\title{
ADAM33 Gene Polymorphisms in Chronic Obstructive Pulmonary Disease
}

\author{
S. Pabst ${ }^{1}$, C. Pizarro Touron ${ }^{1}$, A. Gillissen ${ }^{2}$, M. Lennarz ${ }^{1}$, I. Tuleta ${ }^{1}$, G. Nickenig ${ }^{1}$, D. Skowasch ${ }^{1}$, \\ C. Grohé 3 \\ ${ }^{1}$ Medizinische Klinik und Poliklinik II, Department of Medicine, Bonn University Hospital, Germany; \\ ${ }^{2}$ Department of Pneumology, St. George Medical Center, Leipzig, Germany; ${ }^{3}$ Ev. Lungenklinik Berlin-Buch, Germany
}

\begin{abstract}
Study objective: The pathogenesis of chronic obstructive pulmonary disease (COPD) is characterized by an interaction of environmental influences, particularly cigarette smoking, and genetic determinants. Given the global increase in COPD, research on the genomic variants that affect susceptibility to this complex disorder is reviving. In the present study, we investigated whether single nucleotide polymorphisms in 'a disintegrin and metalloprotease' 33 (ADAM33) are associated with the development and course of COPD.

Patients and design: We genotyped 150 German COPD patients and 152 healthy controls for the presence of the F+1 and S_2 SNPs in ADAM 33 that lead to the base pair exchange $G$ to $A$ and $C$ to $G$, respectively. To assess whether these genetic variants are influential in the course of COPD, we subdivided the cohort into two subgroups comprising 60 patients with a stable and 90 patients with an unstable course of disease.

Results: In ADAM33, the frequency of the F+1 A allele was $35.0 \%$ among stable and $43.9 \%$ among unstable COPD subjects, which was not significantly different from the $35.5 \%$ found in the controls $(\mathrm{P}=0.92$ and $\mathrm{P}=0.07$, respectively). The frequency of the $\mathrm{S} \_2$ mutant allele in subjects with a stable COPD was $23.3 \%(\mathrm{P}=0.32)$, in subjects with an unstable course $30.6 \%(\mathrm{P}=0.47)$.

Conclusion: The study shows that there is no significant difference in the distribution of the tested SNPs between subjects with and without COPD. Furthermore, these polymorphisms appear to have no consequences for the stability of the disease course.
\end{abstract}

Key words: COPD, ADAM33, genetics

\section{INTRODUCTION}

Progressive airflow limitation due to chronic obstructive bronchitis and emphysema is the main characteristic of chronic obstructive pulmonary disease (COPD). At the moment, COPD ranks fourth as a global cause of death and shows a worldwide increase both in morbidity and mortality. This development has provoked rising interest in the clarification of the pathogenic mechanisms underlying this common disease, in order to deduce appropriate and effective therapeutic interventions. Environmental and genetic determinants and their interactions influence COPD susceptibility. The most significant environmental contributor is tobacco smoke. However, not all smokers develop COPD, indicating that genetic factors are at play. Genomic approaches have investigated multiple candidate genes, with inconsistent results [1]. Research on the genetic bases for COPD is therefore still required.

A genetic study has identified ADAM33 to be a susceptibility gene for asthma [2]. It codes for 'a disintegrin and metalloproteinase', a subfamily of transmembrane metalloproteinases, and it shows preferential expression in lung fibroblasts and airway smooth muscle cells. The ADAM33 gene is a putative gene for airway remodeling, as correlations between polymorphisms in ADAM33 and asthma affect both the disease development and its progression.

To determine whether SNP's in this gene predict the progression of COPD in a comparable manner, we examined two polymorphic variants ( F+1 and S_2) that have previously been described to affect the course of asthma [3, 4] and that have been shown to influence the decline of lung function in a Dutch cohort [5].

\section{Material AND Methods}

\section{SAMPLES}

One hundred and fifty German subjects with a clinical diagnosis of chronic obstructive lung disease following the GOLD (The Global Initiative for Chronic Obstructive Lung Disease) guidelines of the ATS/ERS participated in the study. This COPD group was subdivided in 60 patients with a stable course, defined as less than three hospitalizations over the last year due to COPD, and 90 patients with instable disease. Patient recruitment was carried out at the Medical Policlinic, Department of Medicine, Bonn University Hospital, Germany and at the Department of Internal Medicine, St. George Medical Center in Leipzig, Germany. 
Table 1. Baseline characteristics of the COPD and control subjects.

\begin{tabular}{l|l|l|l|l}
\hline & All COPD & Stable COPD & Unstable COPD & Control \\
\hline Number & 150 & 60 & 90 & 152 \\
Age & $62.6 \pm 11.4$ & $61.6 \pm 11.7$ & $63.4 \pm 11.2$ & $63.4 \pm 18.2$ \\
Gender (F/M) & $50 / 100$ & $20 / 40$ & $30 / 60$ & $107 / 45$ \\
Pack/years & $31.1 \pm 22.9$ & $28.6 \pm 15.3$ & $32.8 \pm 26.1$ & $18.7 \pm 8.4$ \\
Smokers & $73(49 \%)$ & $33(55 \%)$ & $40(44 \%)$ & $47(31 \%)$ \\
Non-Smokers & $13(9 \%)$ & $2(3 \%)$ & $11(12 \%)$ & $12(8 \%)$ \\
Ex-Smokers & $64(43 \%)$ & $25(42 \%)$ & $39(43 \%)$ & $93(61 \%)$ \\
FEV1 $\%$ pred $)$ & $52.4 \pm 21.1$ & $59.2 \pm 19.4$ & $47.9 \pm 21.0$ & $81.6 \pm 21.5$ \\
FEV1/FVC & $58.6 \pm 14.9$ & $60.3 \pm 14.7$ & $57.4 \pm 14.9$ & $94.0 \pm 12.7$ \\
GOLD I & 19 & 7 & 12 & \\
GOLD II & 38 & 16 & 22 & \\
GOLD III & 80 & 33 & 47 & \\
GOLD IV & 13 & 4 & 9 & \\
\hline
\end{tabular}

Data are means \pm SD.

Table 2. Sequences of primers for genotyped polymorphisms.

\begin{tabular}{l|l|l}
\hline Gene & Polymorphism & Primers \\
\hline ADAM33 & F+1 & $\begin{array}{l}\text { for: 5'-CTGCCACAATGTACAGTTCCAGGT-3' } \\
\text { rev: 5'-GGAGTGGGAATGCTGTATCTATAGC-3' }\end{array}$ \\
\hline ADAM33 & S_2 & $\begin{array}{l}\text { for: 5'-GACTGGGAGGCCACTGGA-3' } \\
\text { rev: 5'-CGCAGACCATGACACCT'TCCT-3' }\end{array}$ \\
\hline
\end{tabular}

Table 3. Restriction enzymes and length of the resulting fragments.

\begin{tabular}{l|l|l|l}
\hline Gene & Polymorphism & Restriction enzyme & Length of restriction fragments \\
\hline ADAM33 & F+1 & BsmB I & $\begin{array}{l}\text { Wild type (allele G): 215bp + 38bp } \\
\text { Mutation (allele A) : 253bp }\end{array}$ \\
\hline ADAM33 & S_2 & Ban I & $\begin{array}{l}\text { Wild type (allele C): 328bp } \\
\text { Mutation (allele G) }: 262 \mathrm{bp}+66 \mathrm{bp}\end{array}$ \\
\hline
\end{tabular}

The control group included 152 ethnically matched healthy volunteers, recruited at the Medical Policlinic, Bonn University Hospital. Both patient and control groups comprised individuals of Caucasian ancestry. Baseline characteristics of the subjects are displayed in Table 1.

Sample collection was approved by the Ethical Committee of the Medical Faculty in Bonn and of St. George Medical Center in Leipzig. Written informed consent was obtained from all participants prior to inclusion into the study. Genomic DNA was extracted from peripheral blood leucocytes by the salting-out procedure described by Miller et al. [6].

\section{Polymerase Chain Reaction (PCR) And}

Restriction Fragment Length Polymorphism (RFLP)

Genotyping for the genetic variants in ADAM33 in the COPD and control cohorts was carried out by means of PCR and RFLP. As described before, PCR was performed in a total volume of $25 \mu$ l containing 1 $\mu \mathrm{g}$ of genomic DNA, 0.5 U Taq polymerase (Invitrogen, Karlsruhe, Germany), $1 \mu \mathrm{l}$ of each $1.25 \mathrm{mM}$ nucleotide ( MWG-Biotech, Ebersberg, Germany), $2.5 \mu \mathrm{l}$ 10 x PCR-buffer (Invitrogen), $2 \mathrm{mM}$ of $\mathrm{MgCl}_{2}$ (Invitrogen), and $1 \mu \mathrm{l}$ of each $10 \mu \mathrm{M}$ primer (MWGBiotech) [7, 8]. Identical primers as described in the study from van Diemen et al. were chosen (Table 2) [5].

PCR was run in a Thermocycler (T-Gradient, Biometra, Goettingen, Germany) with following cycling conditions: For amplification of genomic fragments in ADAM33, 6 min denaturation at $95^{\circ} \mathrm{C}$ preceded $30 \mathrm{cy}-$ cles of $30 \mathrm{~s}$ denaturation at $95^{\circ} \mathrm{C}, 30 \mathrm{~s}$ annealing at $64^{\circ} \mathrm{C}, 1 \mathrm{~min}$ extension at $74^{\circ} \mathrm{C}$, and one last cycle of extension for $10 \mathrm{~min}$ at $74^{\circ} \mathrm{C}$. F+1 and S_2 SNP genotyping was performed by digestion with the restriction endonucleases BsmB I (New England Biolabs, Frankfurt am Main, Germany) and Ban I (New England Bi- 
olabs), respectively. The resulting fragments were separated on 3\% MetaPhor agarose (Biozym, Hessisch Oldendorf, Germany) and stained with ethidium bromide (Table 3).

\section{STATISTICAL ANALYSIS}

Statistical analysis was performed using software SPSS (version 13.0, Chicago, IL, USA). For each SNP, the distribution of genotypes was tested for differences between controls and stable/instable patients using Armitage's trend test in accordance with the guidelines given by Sasieni [9]. The number of participants in the study led to a statistical power that ranged above 0.8 .

\section{RESULTS}

Results of genotyping are summarized in Tables 4 to 5. In detail, we observed the following constellations. In ADAM33, the distribution of the F+1 locus SNP in the COPD population did not differ substantially from that obtained in the control group. Among the healthy subjects $(n=152)$, the frequency of the $F+1$ rare allele was $35.5 \%$ ( 74 heterozygotes and 17 homozygotes for mutation); in individuals with COPD $(\mathrm{n}=150)$, the frequency was $40.3 \%$ ( 73 heterozygotes and 24 homozygotes for mutation, $\mathrm{P}=0.22$ ). The comparison of the stable and unstable COPD patients with the healthy controls revealed a frequency of the $\mathrm{F}+1$ mutant allele of $35 \% \quad(\mathrm{P}=0.92)$ and $43.9 \%$ $(\mathrm{P}=0.07)$, respectively.

With regard to the S_2 SNP, 72 out of the 152 controls were heterozygous and 6 homozygous for the polymorphism (mutant allele frequency: 27.6\%). In individuals with COPD, 61 were heterozygous and 11 homozygous (mutant allele frequency: $27.7 \%, \mathrm{P}=$ 0.99). In an additional analysis, 27 of the 60 stable COPD patients were carriers of the rare allele for S_ 2 (frequency: $23.3 \%, \mathrm{P}=0.32$ ); among 90 COPD patients with instable course, $30.6 \%$ carried the mutant allele $(P=0.47)$. Similarly to $F+1$, we found no significant difference in distribution of $\mathrm{S} \_2$, neither in the complete COPD collective nor in the subpopulations.

With a significance level set to $\mathrm{P}>0.05$, the examined SNPs were in Hardy-Weinberg equilibrium in the different populations studied. Merely the ADAM33 S_2 polymorphism showed a borderline pertinence in the control group, but a minor allele frequency of $27.6 \%$ was in a range of frequencies obtained in other studies investigating comparable healthy populations (25\% and $24.1 \%$ in the studies by Simpson et al. [4] and van Diemen et al. [5], respectively). Using Armitage's trend test for statistical analysis of genotype distribution, we additionally employed a method characterized by a higher robustness against departures from assumptions of Hardy-Weinberg equilibrium.

Table 4. Case-control analysis of ADAM33 F+1 allele frequencies and genotype distributions (genotypes in comparison with controls)

Homozygotes for wild type

Heterozygotes for mutation

Homozygotes for mutation

Carrier frequency of the mutant allele

P-value (Armitage's trend test)

\begin{tabular}{|l|l|l|l|}
\hline All COPD & Stable COPD & Unstable COPD & Control \\
\hline 53 & 24 & 29 & 61 \\
$(35.3 \%)$ & $(40.0 \%)$ & $(32.2 \%)$ & $(40.1 \%)$ \\
73 & 30 & 43 & 74 \\
$(48.7 \%)$ & $(50.0 \%)$ & $(47.8 \%)$ & $(48.7 \%)$ \\
24 & 6 & 18 & 17 \\
$(16.0 \%)$ & $(10.0 \%)$ & $(20.0 \%)$ & $(11.2 \%)$ \\
$40.3 \%$ & $35.0 \%$ & $43.9 \%$ & $35.5 \%$ \\
$(121 / 300)$ & $(42 / 120)$ & $(79 / 180)$ & $(108 / 304)$ \\
0.22 & 0.92 & 0.07 & \\
\hline
\end{tabular}

Table 5. Case-control analysis of ADAM33 S_2 allele frequencies and genotype distributions (genotypes in comparison with controls).

\begin{tabular}{l|l|l|l|l}
\hline \multirow{2}{*}{ Homozygotes for wild type } & All COPD & Stable COPD & Unstable COPD & Control \\
\cline { 2 - 5 } & 78 & 33 & 45 & 74 \\
Heterozygotes for mutation & $(52.0 \%)$ & $(55.0 \%)$ & $(50.0 \%)$ & $(48.7 \%)$ \\
& 61 & 26 & 35 & 72 \\
Homozygotes for mutation & $(40.7 \%)$ & $(43.3 \%)$ & $(38.9 \%)$ & $(47.4 \%)$ \\
Carrier frequency of the mutant allele & 11 & 1 & 10 & 6 \\
P-value (Armitage's trend test) & $(7.3 \%)$ & $(1.7 \%)$ & $(11.1 \%)$ & $(4.0 \%)$ \\
\hline
\end{tabular}




\section{DisCUSSION}

To demonstrate the evidence for the association of genetic variations within ADAM33 regarding progression of COPD, we genotyped two SNPs in ADAM33. The study was performed in a Caucasian population of COPD patients by means of a case-control approach. However, on the basis of our data, we were not capable of showing significant distributive differences of the tested polymorphisms between German COPD individuals and matched healthy controls neither in a total patient population sample, nor in subdivisions in accordance with the disease stability of course.

ADAM proteins are transmembrane, zinc-dependent metalloproteases with various biological functions, encompassing signal transduction and cell fusion and communication. ADAM33, a member of this gene family, has attracted attention as a possible contributor to asthma development. By using positional cloning techniques, van Eerdewegh et al. [2] have identified ADAM33, encoded on chromosome 20p13, as a susceptibility gene for asthma. Additionally, they demonstrated that ADAM33 is expressed in airway smooth muscle cells and lung fibroblasts, but not in the bronchial epithelium. Given this pattern of expression and the ability of ADAM family members to release growth factors and cytokines, it seems biologically plausible that ADAM33 has a role in airway remodeling as observed in asthmatics. This has been proven recently [10]. Some case-control and family linkage studies have found an association between single nucleotide polymorphisms in ADAM33 and asthma. However, the associated SNPs varied between the populations studied, indicating that the functionally relevant mutation in ADAM33 has not yet been established. For our research, we evaluated a subset of SNPs that has previously been found to modify the course of asthma rather than its onset: The F+1 locus SNP has been demonstrated to be the one that, in early life, is associated with lung function loss at both 3 and 5 years of age [4]. Another study detected an association between the S_2 polymorphism in exon S and functional respiratory impairment in Dutch asthma patients [3]. Additional evidence for ADAM33 as a contributor to accelerated airway dysfunction in another obstructive and inflammatory pulmonary disease as asthma has been provided recently [11]. A correlation between ADAM33 expression levels and asthma severity and changes in FEV1 was described. In consideration of these outcomes, we focused on the gene's role in the progression of airway diseases.

However, approaches to clarify whether these results are transferable to the pathogenesis and pathophysiology of COPD are rare. Apart from chronic obstructive bronchitis, emphysema is a main pathological condition for COPD [12]. An imbalance of endogenous proteinases and antiproteinases is considered to be a major mechanism for emphysematous lung destruction. In this context, matrix metalloproteinases have aroused interest due to their capability of inducing emphysema by proteolysis of lung parenchyma. Similar to matrix metalloproteinases, the ADAM family possesses proteolytic and adhesive activity. Alterations in ADAM33 as a member of this gene family may therefore affect the regulation of cell-cell and cell-matrix adhesion and the extent of extracellular matrix degradation. So it is conceivable that mutations in ADAM33 might promote emphysematous changes, a main feature of COPD. Studies on the distribution of ADAM33 indicate a selective expression in mesenchymal cells, such as lung fibroblasts and bronchial smooth muscle, but not in $\mathrm{T}$ cells or inflammatory leukocytes [13]. This is interesting, as there is evidence, that the homozygotic minor variants of SNP S_2 lead to lowered CD8 cell numbers in patients compared with the wild type [14]. In COPD the predominant cells are macrophages, $\mathrm{T}$ lymphocytes and neutrophils. Cigarette smoke may induce macrophages to release neutrophil-chemotactic factor; thereupon, stimulated macrophages and neutrophils may provoke the release of proteinases. To provide insights into the importance of ADAM33 polymorphisms to the development and course of COPD, further population and functional studies are required.

In the already mentioned Dutch general population study, eight SNPs in ADAM33 in a 1,390 subjects were genotyped [5]. In the general population, a significant excessive decline in FEV1 in homozygotes for the rare alleles of SNPs S_2 was observed; baseline FEV1, however, showed no association to any of the tested polymorphisms. Additionally $\mathrm{F}+1$ and $\mathrm{S} \_2$ were significantly higher prevalent among the subgroup of 186 COPD patients. We only found a trend in F+1 SNP associated to an instable course of COPD. These observations suggest importance of ADAM33 both to COPD development and to its course, reflected in respiratory function decline.

As case-control researches are particularly susceptible to population stratification, a major source of bias, we adjusted our study for ethnic background to reduce this potential confounder: Both the patient and the control cohort solely included individuals of German Caucasian ancestry that presented no migration influences. However, in opposition to the Dutch results, our data suggest that the polymorphisms studied have no impact on the onset of COPD or on its progression. This fact might be due to sample size or different genetic background, even in genetically quite similar populations, such as the German and the Dutch.

Another recently published study examined 25 SNPs of ADAM33 in 880 smokers. A significant association between 5 SNPs and a COPD was found. S_2 also was among them. But linkage disequilibrium and haplotype analyses suggested that S_1 had the strongest degree of association with COPD and pulmonary function abnormalities [15]. Therefore in ADAM33 other SNPs than S_2 and F+1 might lead to a higher susceptibility toward COPD.

In conclusion, our results show no genetic association between polymorphic variants in ADAM33 and the onset and course of COPD. As often seen in genetic case-control studies, data are contradictory in different samples, even if they are all Caucasians. The study of single genes cannot reflect the complexity of COPD pathogenesis. For that reason, the involvement of ADAM33 in COPD development cannot be completely excluded. To estimate their true effect size, further approaches with enlarged sample sizes in differ- 
ent ethical backgrounds and functional studies may improve understanding of the underlying pathogenetic mechanisms and the influence of ADAM33.

Acknowledgements: The authors wish to thank all patients in the present study for their participation. Especially, we thank the staff of the participating hospitals that helped in patient recruitment and sample collection. We would also like to thank Prof. T. Wienker of the Department of Genetic Epidemiology, Bonn University for his help in statistical analysis. This study was supported by institutional grants (BONFOR).

Conflicts of interest: There was not any personal or financial support or author involvement with organizations with financial interest in the subject matter and that effect concerns all the authors of this article.

\section{REFERENCES}

[1] Lomas DA, Silverman EK. The genetics of chronic obstructive pulmonary disease. Respir Res 2001; 2: 20-6.

[2] Van Eerdewegh P, Little RD, Dupuis J, Del Mastro RG, Falls K, Simon J, Torrey D, Pandit S, McKenny J, Braunschweiger K, Walsh A, Liu Z, Hayward B, Folz C, Manning SP, Bawa A, Saracino L, Thackston M, Benchekroun Y, Capparell N, Wang M, Adair R, Feng Y, Dubois J, FitzGerald MG, Huang H, Gibson R, Allen KM, Pedan A, Danzig MR, Umland SP, Egan RW, Cuss FM, Rorke S, Clough JB, Holloway JW, Holgate ST, Keith TP. Association of ADAM33 gene with asthma and bronchial hyperresponsiveness. Nature 2002; 418: 426-30.

[3] Jongepier H, Boezen HM, Dijkstra A, Howard TD, Vonk JM, Koppleman GH, Zheng SL, Meyers DA, Bleecker ER, Postma DS. Polymorphisms of the ADAM33 gene are associated with accelerated lung function decline in asthma. Clin Exp Allergy 2004; 34: 757-60.

[4] Simpson A, Maniatis N, Jury F, Cakebread JA, Lowe LA, Holgate ST, Woodcock A, Ollier WER, Collins A, Custovic A, Holloway JW, John SL. Polymorphisms in a disintegrin and metalloprotease 33 (ADAM33) predict impaired early-life lung function. Am J Respir Crit Care Med 2005; 172: 55-60.

[5] Van Diemen CC, Postma DS, Vonk JM, Bruinenberg M, Schouten JP. A disintegrin and metalloprotease 33 polymorphisms and lung function decline in the general population. Am J Respir Crit Care Med 2005; 172: 329-33.

[6] Miller SA, Dykes DD, Polesky HF. A simple salting out procedure for extracting DNA from human nucleated cells. Nucleic Acids Res 1988; 16: 1215.
[7] Pabst S, Yenice V, Lennarz M, Tuleta I, Nickenig G, Gillissen A, Grohé C. Toll-like receptor 2 gene polymorphisms Arg677Trp and Arg753Gln in chronic obstructive pulmonary disease. Lung 2009; 187: 173-8.

[8] Vacca G, Schwabe K, Dück R, Hlawa HP, Westphal A, Pabst S, Grohé C, Gillissen A. Polymorphisms of the beta2 adrenoreceptor gene in chronic obstructive pulmonary disease. Ther Adv Respir Dis 2009; 3: 3-10.

[9] Sasieni PD. From genotypes to genes: doubling the sample size. Biometrics 1997; 53: 1253-61.

[10] Puxeddu I, Pang YY, Harvey A, Haitchi HM, Nicholas B, Yoshisue H, Ribatti D, Clough G, Powell RM, Murphy G, Hanley NA, Wilson DI, Howarth PH, Holgate ST, Davies DE. The soluble form of a disintegrin and metalloprotease 33 promotes angiogenesis: implications for airway remodeling in asthma. J Allergy Clin Immunol 2008; 121: 1400-06.

[11] Lee JY, Park SW, Chang HK, Kim HY, Rhim TY, Lee JH, Jang AS, Koh ES, Park CS. A disintegrin and metalloproteinase 33 protein in asthmatics: relevance to airflow limitation. Am J Respir Crit Care Med 2006; 173: 729-735.

[12] Barnes PJ. Chronic obstructive pulmonary disease. N Engl J Med 2000; 343: 269-80.

[13] Yoshinaka T, Nishii K, Yamada K, Sawada H, Nishiwaki E, Smith K, Yoshin K, Ishiguro H, Higashiyama S. Identification and characterization of novel mouse and human ADAM33s with potential metalloprotease activity. Gene 2002; 282: 227-36.

[14] Gosman MM, Boezen HM, van Diemen CC, SnoeckStroband JB, Lapperre TS, Hiemstra PS, Ten Hacken NH, Stolk J, Postma DS. A disintegrin and metalloprotease 33 and chronic obstructive pulmonary disease pathophysiology. Thorax 2007; 62: 242-7.

[15] Sadeghnejad A, Ohar JA, Zheng SL, Sterling DA, Hawkins GA, Meyers DA, Bleecker ER. ADAM33 polymorphisms are associated with COPD and lung function in long-term tobacco smokers. Respir Res 2009; 10: 21.

\author{
Address for correspondence: \\ Dr. med. Stefan Pabst \\ Medizinische Klinik und Poliklinik II \\ Universitätsklinikum Bonn \\ Sigmund-Freud-Str. 25, \\ 53105 Bonn \\ Germany \\ Phone: $\quad+4922828715259$ \\ Fax: $\quad+4922828716980$ \\ E-mail: Stefan.pabst@ukb.uni-bonn.de
}

